\title{
LA LABOR DE ENRIC GRANADOS EN EL PROCESO DE LA RECEPCIÓN DE LA MÚSICA DE BACH EN BARCELONA
}

\author{
Francesc BONASTRE \\ Universitat Autònoma de Barcelona
}

\begin{abstract}
The musical personality of Enric Granados (1867-1916) includes many aspects, especially piano interpretation and symphonic, scenic, "liederistic" and piano composition, as well as pedagogy, together with the foundation of the Granados Academy (1900). He got to know Bach's music through Ch. W. Bériot in Paris and F. Pedrell in Barcelona. He spread Bach's music as a soloist, director and pedagogue, together with other important disciples of Pedrell: J. Albéniz, L. Millet and H. Anglès. The article includes 14 concerts in Barcelona related to Granados (1895-1912).
\end{abstract}

\section{Resumen}

La personalidad musical de Enric Granados (1867-1916) comprende numerosas facetas, especialmente la interpretación pianística, la composición sinfónica, escénica, liederística y pianística, y la pedagogía, con la fundación de la Academia Granados (1900). Conoce la música de Bach a través de Ch. W. Bériot en París, y de F. Pedrell en Barcelona; se convierte en uno de los difusores de la música bachiana como concertista, director y pedagogo, al lado de otros destacados discípulos de Pedrell: I. Albéniz, L. Millet e H. Anglès. El artículo recoge 14 conciertos barceloneses relacionados con Granados (1895-1912).

La lenta recuperación de la vigencia estética de la música de J.S. Bach iniciada en la primera mitad del siglo XIX, cambia de registro en la segunda mitad de la centuria, debido a múltiples causas, las más importantes de las cuales podrían ser, a mi juicio, dos: en primer lugar, el despliegue editorial iniciado en 1851 con la edición programada de las obras completas a cargo de la Bach Gesellschaft (BGA, Bach-Gesellschaft Ausgabe) en Leipzig (y su reflejo en la competencia con diversas editoriales europeas que publicaron parte de la obra bachiana, y por otro lado, la respuesta creciente de los intérpretes, que incorporan a su repertorio de música coetánea algunas de las obras del maestro de Eisenach.

La Barcelona de esta época se halla en un estadio de abatimiento musical, a excepción del medio operístico, que desde la erección del teatre del Liceu en 1847 recupera la atención de la burguesía y de la alta sociedad urbana; ello no obstante, no hay que olvidar que la traumática abolición del Antiguo Régimen a finales de la primera mitad del siglo supuso, desde el punto de vista 
musical, la desaparición de muchos puestos de trabajo, y de bastantes capillas musicales de las más de veinticinco existentes en Barcelona antes de esta circunstancia. ${ }^{1}$

Por todo ello, tuvo que ser la propia sociedad civil la que tomó paulatinamente la responsabilidad de una educación y de una cultura públicas, reiniciando un proceso de recepción que se había interrumpido justo al inicio del romanticismo musical.

No es de extrañar, pues, que junto a esta renovada toma de contacto con la gran música europea, Barcelona recuperara a la vez no sólo la música sinfónica, camerística y operística internacional, sino también las adherencias que ésta había provocado (especialmente en el área germánica, pero también en la francesa e italiana), cuya máxima representatividad residía en el resurgir de la figura emblemática de Juan Sebastián Bach. El hecho es perfectamente conocido y ha sido suficientemente glosado por los estudiosos; señalemos, no obstante, que el problema de la disfunción entre música y estética (y entre estética de la producción y estética de la recepción) no afectaba sólo a Bach, sino a la fortuna de todos los compositores de su época y de las anteriores, hasta la desaparición del Antiguo Régimen. La presencia de su música junto al despertar de las primeras producciones románticas se convierte en un proceso de abstracción: rodeado de un hálito de prestigio, se coloca a Bach no sólo como la cúspide de la música de su tiempo (cuya entidad era aún desconocida), sino como antecesor del futuro, como alfa y omega de la música occidental.

Las primeras obras de Bach que aparecen en los documentos históricos de los archivos referidos a los conciertos de Barcelona, datan de 1866, primero de manera esporádica, y luego con un progresivo crecimiento hasta 1900 , el repertorio bachiano aumenta espectacularmente en la primera década del siglo XX y duplica su número en la segunda, para culminar con el estreno de la Matthäus-Pasion en $1921^{2}$ : a partir de esta fecha, la música de J.S. Bach entra de lleno en el canon musical de los conciertos barceloneses.

Una de las personalidades que contribuyó a este proceso fue Enric Granados (1867-1916), que con Isaac Albéniz (1860-1909) configuró la primera promoción de discípulos de Felip Pedrell, hacia 1883-1884³ ; el contacto con el maestro se mantuvo durante toda su vida, y Pedrell tuvo que pasar por la desgracia de ver morir en plena juventud a estos primeros frutos de su maestrazgo musical; si bien Granados, Albéniz y Falla siempre se reconocieron como discípulos, la actitud de Pedrell es más sensible al talento de los que recibieron sus enseñanzas. A la muerte de Granados, y ante determinadas afirmaciones en relación con la influencia de Scarlatti y Schumann sobre su personalidad musical, puntualiza:

1. BonAStRE, Francesc: "L'Oratori a Barcelona en el primer terç del segle XIX”, Anuario Musical, 48 (1993), $207-216$.

2. ID.: La recepció de la música de Bach a Barcelona (1866-1921. Barcelona, Biblioteca de Catalunya, 2002 (en premsa). Cf. también Id., "Els inicis de la recepció de la música de J.S.Bach a Barcelona", Revsta Musical Catalana, 193 (noviembre de 2000), 6-7.

3. Hess, C.: Enrique Granados. A Bio-Bibliography. Greenwood Press, New York, 1991,p.6. Cf. también SALvat, J.: "Enric Granados. Notes biogràfiques", RMC, XIII (15 de juny de 1916), 198. 
"Tinc altres idees sobre això. I les tinc, per haver secundat sa gran facultat d'assimilació, guiant-lo, conduint-lo, menant-la mitjançant diversos intents; perquè no puc ni sé tenir la tonta vanitat d'afirmar que l'he ensenyat; com no la tinc tampoc dient, per exemple, que he ensenyat a En Falla, a N'Albèniz i a tutti quanti que figuren en la nombrosa llista de ma família espiritual artística. A En Granados, com a tots els demés que s'hi prestaven, els he guiat, simplement, per sí, perquè tenien aquella qualitat per sí que solament posseeixen els artistes elegits". ${ }^{4}$

Granados amplió sus estudios musicales en París, siguiendo el consejo de Pedrell: desde septiembre de 1887 hasta julio de 1889 residió en la capital francesa, donde recibió clases de piano de Charles Wilfrid de Bériot; entre los modelos de la música que dicho profesor recomendaba para este instrumento estaba J.S. Bach, de quien aconsejaba especialmente el estudio de sus Preludios y Fugas, que él mismo interpretaba ante sus alumnos con frecuencia; asimismo, dedicaba un día a la semana al estudio facultativo de la música antigua de tecla. ${ }^{5}$

Este primer conocimiento de Bach y de su contexto que Granados recibió en París en las clases de Bériot, se vio acrecentado por la idea regeneradora del nacionalismo musical que Pedrell publicó en el manifiesto Por nuestra música, en 1891, después de haber compuesto la trilogía Els Pirineus, con texto de Víctor Balaguer. En su tratado, Pedrell habla de Bach como "Palestrina del Norte", refiriéndose a la misión forjadora de la música artística, como ejemplificación de la segunda fase del canto nacional y como muestra del impulso de la consciencia musical germánica. ${ }^{6}$ Este primer esbozo del valor de la música bachiana -referida especialmente al trabajo contrapuntístico de los temas de los corales, tomados éstos como expresión del ancestral Volkgeist- lo amplía en su discurso de ingreso en la Real Academia de Bellas Artes de San Fernando de Madrid, aduciendo el testimonio del musicólogo alemán Carl Krebs (1857-1937): según éste, existía una afinidad de concepto entre las Salmodias, Tientos e Interludios de Cabezón y los Corales de J.S. Bach para órgano, ${ }^{7}$ con lo cual Pedrell intenta universalizar los fenómnos de aculturación musical, guardando las diferencias de planteamiento, método y finalidad, manteniendo sólo "el punto de vista de la idealidad artística". ${ }^{8}$

La visión pedrelliana de Bach no sólo se refirió al concepto, sino especialmente a la vertiente práctica. Era preciso que el público se percatara exactamente del valor de la música de Bach, aparecida tardíamente y fuera de su contexto: por ello dice, muy gráficamente,

"Se le han dado al público platos fuertes sin entremeses y, dígase lo que se quiera, se le ha indigestado la alimentación, demasiado nutritiva, subiéndosele a la cabeza los gases de una digestión mal hecha.... Sin un buen régimen curativo de vulgarización, que a la par que aumente sus

4. Pedrell, F.: "La personalitat artística d'En Granados", RMC, XIII (15 de juny de 1916), 173-174.

5. Bergadè Armengol, M.: Les pianistes catalans à Paris entre 1875 et 1925. Contribution à l'étude des relations musicales entre la France et l'Espagne. Tesis doctoral defendida en la Université François Rabelais de Tours (Francia), UFR de Musique et Musicologie, en enero de 1997. Capítulo 2:"L'autorité du Conservatoire", págs. 97-100.

6. Pedrell, F.: Por nuestra Música... Barcelona, Heinrich i Cia., 1891.

7. ID.: Discurso leído ante la Real Academia de Bellas Artes de San Fernando en la recepción pública del Sr. D..., el día 10 de marzo de 1895. Publicado también en fascículos en La llustración Musical Hispano-Americana ; la cita corresponde al número 177 de la citada IMHA (30 de mayo de 1895), 265, col. a.

8. ID., Ibid., pág. 265 , col. c. 
goces artísticos, eleve su capacidad intelectual hasta el extremo de saber oir música, arte que no posee ahora, es de temer que el público de nuestros conciertos... muera de empacho de música, de música de última hora."9

Al reclamo de esta "vulgarización", es decir, de actividad concertística y pedagógica para dar a conocer adecuadamente la música, acudieron todos sus discípulos. En este aspecto, la labor de Granados queda recogida en dos tipos de actividades: la concertística, tanto en lo que se refiere a la interpretación pianística como a la labor directorial y la pedagógica.

En primer lugar, observamos el protagonismo de Granados como pianista en los 14 conciertos que tienen lugar el Barcelona desde 1895 hasta 1912. Las obras que interpreta (como protagonista, como acompañante o como director) pueden ser identificadas casi todas:

1. "Concert per a dos violins amb acompanyament de piano" ${ }^{10}$ (conciertos núms. 1 y 3 del apéndice documental, así como el "Concert per a dos violins" del núm. 10); se trata del Concierto en Re menor para dos violines, cuerda y continuo, BWV 1043, en su versión simplificada para dos violines solistas y piano. Fue publicada en la $B G A$, vol. XXI, por W. Rust en 1874.

2. "Trio en Sol major, per a piano, flauta i violi"” (concierto núm. 4): es la Sonata en Sol mayor BWV 1038/ Ahn. II 154, hoy atribuída a un hijo de Bach o a un discípulo, pero que en aquella época formaba parte de la tradición bachiana. Fue publicada en la $B G A$, vol. IX, por W. Rust en 1860.

3. "Fuga en Do sost[ingut] menor per a flauta, oboè, clarinet, dos fagots, trompa $i$ orquestra de corda" Se trata de una instrumentación de la Fuga en Do\# menor del I Volumen del Das Wohltemperierte Klavier, BWV 849/4. Publicado en la BGA, vol. XIV, por F. Kroll en 1866,

4. "Concert en Re menor per a piano i orquesta de corda" (concierto núm. 5): es el Concerto solo, una oboe, due violini, viola e continuo, BWV 1059. Fue publicado en la $B G A$, vol. XVII, por W. Rust en 1869.

5. "Concert en re menor, per a tres pianos amb acomp[anyament] d'orquestra" (concierto núm. 6): es el Concierto en Re menor para tres clavicémbalos, 2 violines, viola y continuo, BWV 1063. Fue publicado en la $B G A$, vol. XXXI, por P. Graf Waldersee, en 1885. Antes había sido pubicado por la Peters en 1846.

6. "Sarabanda (de la $1^{a}$ Partita per a piano)" (conciertos núm. 7, 8 y 9): corresponde a la Partita I en Si menor para clave, BWV 825/4. Fue publicada en la BGA, vol. III, por C.F. Becker, en 1853. Numerosas ediciones anteriores, desde 1801 a 1844.

7. "Fantasia cromàtica i fuga" (id. anterior): es la Chromatische Fantasie und Fugue dmoll, BWV 903. Fue publicada en la $B G A$, vol. XXXVI, por E. Naumann, en 1890. Otras ediciones anteriores, desde 1802 a 1857.

9. ID.: "Programas de conciertos", publicado en La Vanguardia el 31 de marzo de 1902, e incluído en Musicalerías. Selección de artículos escogidos de crítica musical. Sempere y Compañía, Editores. Valencia-Madrid, s.d.[1906]. La cita corresponde a la pág. 20.

10. Tomamos los títulos de las obras de la referencia original del Dietari de Concerts de Barcelona, vol. I (17971900) y vol. II (1900-1920) del IDIM Josep Ricart i Matas. 
8. "Sarabande et Gigue per a violí sol" (conc. núm. 10). Posiblmente se trate de la Partita II en Re menor para violín solo, BWV 1004/3, 4. Fue publicada en la $B G A$, vol. XXVII por A. Dörffel en 1879. Otras ediciones anteriores en 1798 (París), 1802 (Bonn) y 1843 (Leipzig).

9. "Ària (corda sola)" (conc. núm. 11). Por el contexto del Dietari de Concerts de Barcelona antes citado en la nota 9, es muy probable que corresponda a la Ouvertüre D-dur, BWV 1068/2. Fue publicada en la $B G A$, vol. XXXI, por A. Dörffel en 1885. Otras ediciones anteriores en Leipzig, 1817 y 1854.

10. "Sonata III en Mi major per a violí i piano" (conc. núm. 12). Es la Sonata III para violín y clave en Mi mayor, BWV 1016. Fue publicada en la BGA, vol. IX, por W. Rust en 1860. Otras ediciones anteriores desde 1802 a 1841.

Hemos referenciado sólo aquellos conciertos públicos en los que Granados toma parte directamente; como es sabido, la labor de nuestro compositor en la Barcelona de final de siglo es muy densa; desde su colaboración con las instituciones ciudadanas (sobre todo, con la Societat Catalana de Concerts i la Filharmónica) hasta la creación de una serie propia de conciertos (Concerts Clàssics, 1900) y de una orquesta (Orquestra de Barcelona), su protagonismo como solista de piano y como compositor dramático va en aumento y cobra una dimensión internacional. La Academia Granados, fundada en 1900, acoge también la música de Bach, no sólo en los programas pedagógicos y en los conciertos, sino en cursos especiales como el que dio Pedrell en 1906.

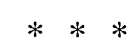

La labor de propagación del repertorio bachiano llevada a cabo por Enric Granados se inscribe dentro del ideario pedrelliano antes aludido; en él colaboraron, de manera muy destacada, otros discípulos del maestro tortosino: destacamos de una manera especial a Isaac Albéniz, que en la Exposición Universal de 1888 dio a conocer el Concerto italiano (BWV 971); a Lluís Millet quien, habiendo fundado el Orfeó Català en 1891, lo convirtió en el instrumento idóneo para la recepción de la música vocal bachiana ${ }^{11}$, y finalmente, a Higini Anglès, que formó parte del comité internacional de la Neue Bach Ausgabe, con el Bach-Institut de Göttingen y el de Leipzig, desde 1955 hasta su muerte.

$$
* * *
$$

Al Prof. Dr. José Vicente González Valle, sucesor de Anglés, dedico cordialmente esta pequeña colaboración, en el hilo conductor del amor bachiano que nos une. Y de tantas otras cosas. Ad multos annos vivas!

11. Bonastre, F.: “La Schola Cantorum de Paris et la réception de la musique de J.S. Bach à Barcelone”. Les rencontres de Villecroze, 1998: Échanges musicaux franco-espagnols, XVIIe-XIXe siècles. Villecroze, 2000, p. 319-334. 


\section{APÈNDICE DOCUMENTAL}

1895 - 13 d'octubre - Societat Catalana de Concerts (Teatre Líric)

$6^{a}$ serie. $1^{\text {a }}$ sesión de música de cámara

Cuarteto Belga de Bruselas

Enric Granados, piano

I

Cuarteto en Fa m, núm. 11, op. $95 \quad$ L.V. Beethoven

II

Suite para violoncelo

D. Zipoli

Concierto para dos violines $y$

acompañamiento de piano

J.S. Bach

III

Cuarteto núm. 2 en Re

A.Borodin

1897 - 26 de abril - Societat Catalana de Concerts

$9^{\mathrm{a}}$ serie. Sesiones privadas

$5^{\circ}$ concierto, $2^{\mathrm{a}}$ sesión de música de cámara

M. Crickboom, L. Angenot, N.Lejeune, H. Gillet, cuarteto

Enric Granados, piano

I

Cuarteto XXI, en Si M

W.A. Mozart

II

Sonata para viola

J.S. Bach

III

Quinteto para piano e

instrumentos de cuerda

C. Franck

3

1897 - 3 de mayo - Societat Catalana de Concerts

$9^{\mathrm{a}}$ serie. Sesiones privadas

$6^{\circ}$ concierto, $3^{\mathrm{a}}$ sesión de música de cámara 
M. Crickboom, L. Angenot, Lejeune, H. Gillet, cuarteto

Enric Granados, piano

$\mathrm{I}$

Cuarteto en Fa M núm. 1, op. 18

L.V. Beethoven

II

Concierto para dos violines y

acompañamiento de piano

J.S. Bach

III

Cuarteto núm. 2 en Re

A.Borodin

1899 - 22 de octubre - Societat Filharmònica (Sala Estela)

3er año. 12 conciertos.

$1^{\mathrm{a}}$ sesión de música de càmara

M. Crickbom, Meriz, violins; J. Calvera, flauta

Forns, viola; Dini, violoncel

Enric Granados, piano

I

Trio en Sol M, fl, vl, y pf

J.S. Bach

II

Cuarteto en Sol m op. 74 núm. 3

F.J. Haydn

III

Sonata en Do m op. 30 núm. 2, vl y pf L.v. Beethoven

1900 -15 de maig - Societat de Concerts Clàssics (Teatre Líric)

1er concert. Orquestra de la Societat

Joaquim Malats, piano

Enric Granados, director

I

Holberg Suite

II

E. Grieg

Impromtu núm. 3

F. Chopin

Legende

I. Paderewski

Andante

Cramer

Fuga en Do \# $m$

J.S. Bach (instrumentada por E. Granados) 
Minuetto en Re

G.F. Haendel

III

Concierto en $\mathrm{Re}$ m para piano

y orquesta de cuerda

J.S. Bach

6

1900 - 11 de novembre- Societat de Concerts Clàssics (Teatre Novetats)

$2^{\text {on }}$ concert. Concert extraordinari. Orquestra de la Societat

C.G. Vidiella, J. Malats i E. Granados, piano

Domènec Sánchez Deyà, director

\section{I}

Sonata para 2 pianos

W.A. Mozart

Concierto en La m para

piano y orquesta

I. Paderewski

II

Variaciones y fuga

J. Fischhof

Concierto en Fa m para

piano y orquesta

III

F. Chopin

Les Djins

C. Franck

Concierto en Re m para tres

pianos y orquesta

J.S. Bach

1902 - 2 de maig - Orfeó Català - (Plaça de St. Just, 1)

Recital de piano por Enric Granados

Dedicado a los coristas de l'Orfeó Català

I

Sonata núm. 21, op. 53 (La Aurora) L.v. Beethoven

II

Sarabanda (Partita I para clave)

Fantasía cromática y fuga

J.S. Bach

III

Au soir

Papillons noirs

R. Schumann

Id. 
Nocturno en Do \# $m$

Polonesa en Mi $b \mathrm{~m}$

Allegro appassionato
F. Chopin

Id.

C. Saint-Saëns

\section{8}

1902 - 6 de mayo - Associació Wagneriana -(Canuda 4, pral.)

Audición de piano por Enrique Granados

Sonata núm. 21, op. 53 (La Aurora)

Sarabanda (Partita I para clave)

Fantasía cromática y fuga
L.v. Beethoven

J.S. Bach

Id.

\section{9}

1902 - 14 de mayo - Teatre Principal

Repetición del programa número 7

10

1904 - 27 de mayo - Societat Filharmònica (Teatre de les Arts)

$3^{\mathrm{a}}$ sèrie de 3 conciertos. 2on concierto Crickboom

M. Crickboom, E. Ysaye, violines

E. Granados, piano

I

Sonata pera 2 vl y piano

G.F. Haendel

Sarabande y Gigue (vl solo)

J.S. Bach

II

Caprice sur l'Étude en forme

de valse de Saint-Saëns (id.)

Concierto en Re m para dos violines

E. Ysaye

y acompañamiento de piano

III

Sonata en Re m op. 108

para violín y piano

J. Brahms 
1905 - 28 de abril - Cercle de Belles Arts - (Teatre Principal)

Orquesta de Barcelona

Ferran Via, piano; Raquel Martí, arpa

3er y último concierto dirigido por E. Granados

I

Preludio y muerte de Isolda

R. Wagner

L'última llàgrima

M. Marqués

Aria [de la Suite en Re]

J.S. Bach

Murmullos de la selva

R. Wagner

II

La cova del drac

M. Marquès

III

Holberg-Suite

E. Grieg

La cacera (Ferran Via, piano)

F. Mendelssohn

Polonesa fantasia

F. Chopin

Tardor (Raquel Martí, arpa)

A. Thomas

Les Djins (piano y orquesta)

C. Franck

Los Maestros Cantores de Nürnberg

R. Wagner

1910 - 9 de noviembre - Palau de la Música Catalana

Concerts Clàssics. 3er concierto.

Jacques Thibaud, violín; Enrique Granados, piano.

I

Sonata III en Mi M para

violín y piano

J.S. Bach

II

Sonata núm 7 en Do m, op. 30,

para piano y violín

L.v. Beethoven

III

Sonata núm. 2 en Re $m$ (id.)

R. Schumann 
1912 - 4 de febrero - Academia Granados-Sala de conciertos (Av. Tibidabo, 18)

Recital de piano, por Enrique Granados

I

Preludio en Mi b $m$

Sonata en $\mathrm{Fa}$

Sonata Patética op. 13

Los requiebros

Polonesa en Mi $b$ m

Berceuse

Impromptu en La $b$ m

Muerte de Isolda

\section{J.S. Bach}

W.A. Mozart

L.v. Beethoven

II
E. Granados
F. Chopin
R. Schumann
F. Schubert
R. Wagner (arr. F. Liszt)

1912- 3 de marzo - Palau de la Música Catalana

Concerts de Quaresma. ${ }^{\circ}$ concierto

$2^{\circ}$ recital Thibaud- Granados

Jacques Thibaud, violín; Enric Granados, piano

I

Sonata en Si b para violín y piano

W.A. Mozart

II

F. Chopin

Nocturno en Do \# $m$

Id.

Balada en La $b$

[J.S.] Bach

III

Sonata para violín y piano

G. Lekeu 